\title{
Angehörige von Menschen mit Demenz sagen «JA» zur Technik
}

Natascha Radenovic

Technikeinsatz für Menschen mit Demenz gehört zu den umstrittensten ethischen Fragen der Betreuung der Betroffenen. Im Rahmen einer Masterarbeit haben Interviews mit Angehörigen von Betroffenen gezeigt, dass Technik niemals Ersatz für menschliche Nähe, jedoch eine angenehme Abwechslung im Alltag der Menschen mit Demenz und auch der Angehörigen selbst sein kann. Dabei kommt vor allem der individuellen Anpassung wesentliche Bedeutung zu.

\section{Les proches des personnes atteintes de démence disent oui à la technologie \\ L'utilisation de la technique pour les personnes at- teintes de démence fait partie des questions d'éthique les plus controversées en matière de prise en charge des personnes concernées. Dans le cadre d'un travail de Master, des entretiens avec les proches des personnes concernées ont montré que la technologie ne remplacerait jamais la proximité humaine, mais qu'elle pouvait être une distraction agréable dans le quotidien des personnes atteintes de démence et aussi leur proches avec une grande importance accordée à l'adaptation individuelle surtout.}

DOl 10.1024/2297-5160/a000025

eitdem das Domicil Kompetenzzentrum Demenz Bethlehemacker über verschiedene technische Angebote verfügt, ist es in den Medien regelmässig präsent. Die Wohnbereiche der Langzeitpflegeeinrichtung in Bern wurde 2013 entsprechend den Bedürfnissen der Menschen mit Demenz angepasst und umgebaut.

\section{Technikeinsatz trifft auf Medieninteresse}

Oberstes Ziel bei der Planung und Umsetzung des Umbaus war es, den Menschen mit Demenz eine hohe Lebensqualität und positive emotionale Erlebnisse zu ermöglichen. Bei dem innovativen Projekt betraten alle Beteiligten zwangs-

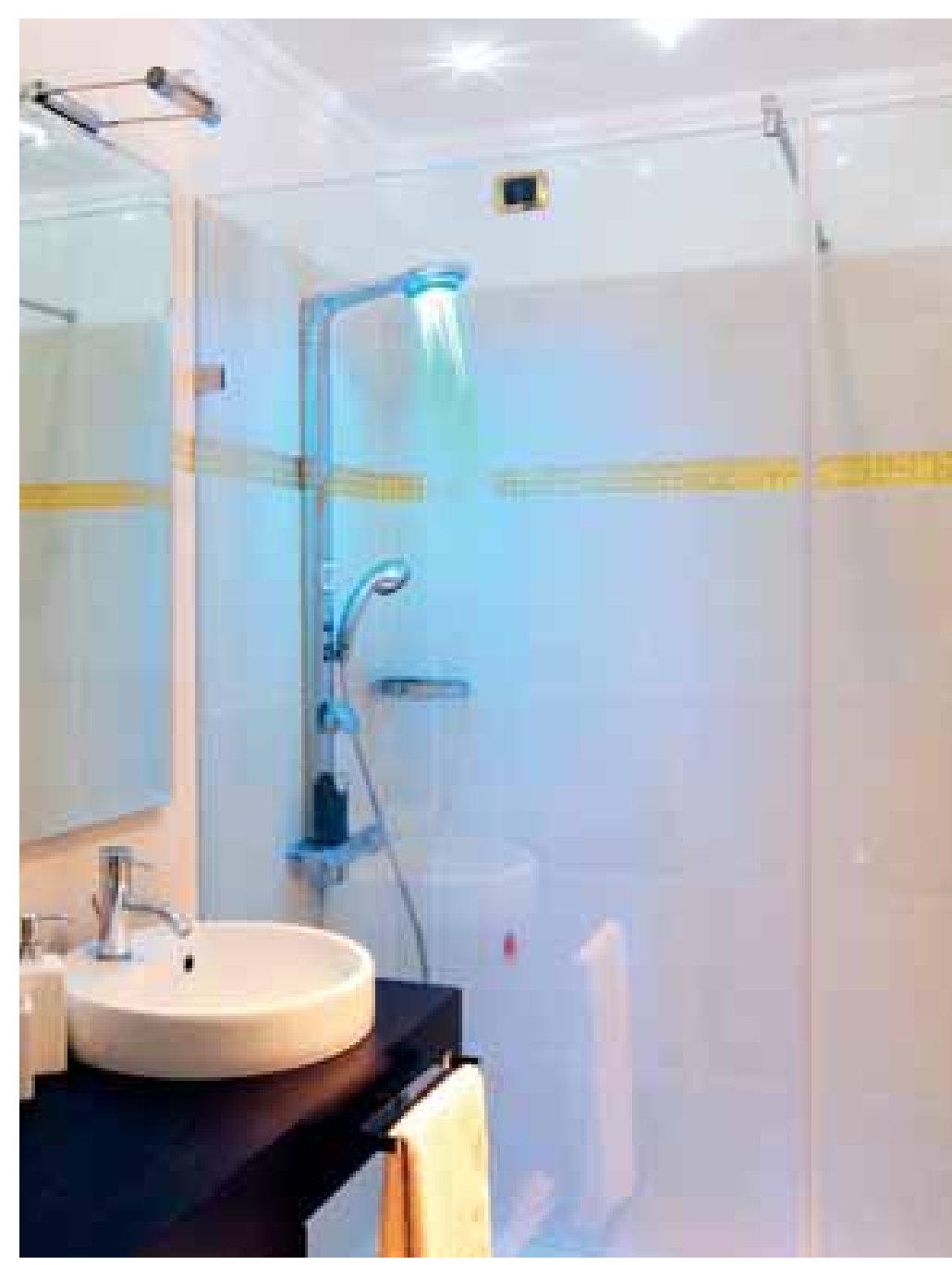

läufig Neuland. Im Fokus ist somit keine Technik zur Sicherheit und Orientierung, sondern es handelt sich dabei um technische Angebote, die der Bewohner mit Demenz nutzen kann, wie z. B. Cheminée/Aquarium als Film in einem Fernseher, Lichtdusche, Massagestuhl, Wasserbett mit Licht, Discokugel, «Wasserfall zum Anfassen» (oder «Spagetti» wie es manche Bewohner nennen), Thermospa, «Blubbersäulen» und ein Zugabteil.

\section{Ethische Fragen}

Die Diskussionen über Anwendung und Nutzung dieser Technik werfen vor allem ethische Fragen auf: Darf dem 
Menschen mit Demenz eine Realität simuliert werden? Welche ethischen Konflikte ergeben sich durch die Nutzung der Technik? Wird durch die Technik die menschliche Nähe ersetzt?

Das Domicil Kompetenzzentrum Demenz Bethlehemacker hat eine Evaluation der Wirkung der Technik zusammen mit der Berner Fachhochschule (BFH) durchgeführt. Eine der Zielgruppen der Evaluation waren die Angehörigen der Menschen mit Demenz. Im Rahmen einer Masterarbeit am Institut Alter der BFH wurden zu drei Erhebungszeitpunkten in Abständen von je sechs Wochen 12 Angehörige zu ihrer Wahrnehmung und Einschätzung des Technikeinsatzes im Rahmen von Interviews befragt.

\section{Angehörige von Menschen mit Demenz im Interview}

Die Auswertung der Interviews zeigte, dass die Angehörigen der Technik offen gegenüberstehen und diese positiv bewerten. Ihrer Meinung nach bringt diese Abwechslung in den Alltag der Menschen mit Demenz, aber auch in jenen der Besucher. Die Angehörigen berichten über eine angenehme und gemütliche Atmosphäre. Sie beobachteten, dass die Bewohner die Technik am liebsten zusammen mit anderen Menschen nutzen. Einige technische Anwendungen können vom Bewohner selber nicht bedient und genutzt werden. Daher werden sie immer von den Angehörigen oder Pflegenden begleitet. Die individuelle, den Wünschen und Vorlieben der Menschen mit Demenz angepasste Nutzung der Technik ist für die Angehörigen wichtig. Sie berichten auch davon, dass sich die Bewohner selber von der Technik entfernen, wenn sie genug davon haben z. B. dann, wenn der Inhalt des Filmes dem Bewohner nicht mehr entspricht.

Die Angehörigen haben aber auch viele gute Ideen zur Nutzung und Optimierung der Technik und ihrer Anwendung geäussert. So beispielsweise, eine einfache Bedienung durch z. B. Start/Stopp-Knopf oder einen Zugang mit Code zu ermöglichen, mit dem der Angehörige die Technik selbstständig bei Besuchen nutzen könnte. Aus den In- terviews wurden, neben den Wünschen der Angehörigen, auch Empfehlungen bezüglich der Technik erschlossen, die im Domicil Kompetenzzentrum Demenz Bethlehemacker als wertvolle Hinweise für Optimierungen genutzt wurden. So wurde beispielsweise vorgeschlagen:

- die Individualität der Bewohnenden mit einem vielseitigeren Angebot an Filmen zu fördern;

- die Nutzung (z.B. Ein-/Ausschalten) der Technikangebote soweit wie möglich zu vereinfachen, damit auch die Angehörigen diese selbstständig bedienen können;

- die Angehörigen besser in die (selbstständige) Nutzung der Angebote einzuführen sowie

- den Austausch zwischen den Angehörigen und Pflegenden bezüglich Technik zu institutionalisieren.

\section{Schlussfolgerungen}

Obwohl der Einsatz von Technik in der Betreuung von Menschen mit Demenz mit vielen ethischen Fragen besetzt ist, haben die Angehörigen von keinen ethischen Konflikte berichtet, weder innerhalb der Institution noch ausserhalb, beispielsweise im Gespräch mit Bekannten oder anderen Personen. Da die Menschen mit Demenz grösstenteils auf menschliche Hilfe angewiesen sind, kann die Technik auf keinen Fall ein Ersatz für menschliche $\mathrm{Zu}$ wendung sein, jedoch eine willkommene und angenehme Unterstützung und Abwechslung im Alltag.

\section{Literatur}

Radenovic, N. (2015) Technik und ihre Auswirkungen auf die Menschen mit Demenz aus der Sicht der Angehörigen. MAS «Gerontologie - Altern: Lebensgestaltung 50+». Institut Alter, Berner Fachhochschule (BFH).

Weiterführende Literatur kann bei der Autorin bezogen werden.

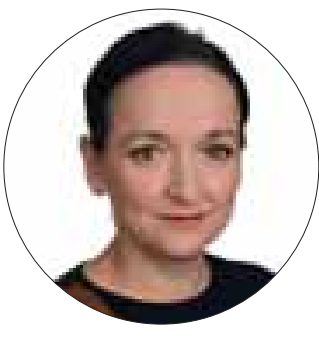

Natascha Radenovic ist Pflege-

expertin und Leiterin Betreuung und

Pflege im Domicil Steigerhubel, Bern.

natascha.radenovic@domicilbern.ch 